\title{
Double parton distributions of the pion in the NJL model
}

\author{
Wojciech Broniowski ${ }^{* \dagger}$ \\ Jan Kochanowski University, 25-406 Kielce, Poland, and \\ Institute of Nuclear Physics PAN, 31-342 Cracow, Poland \\ E-mail: Wojciech.Broniowski@ifj.edu.pl
}

\section{Enrique Ruiz Arriola}

Departamento de Física Atómica, Molecular y Nuclear and Instituto Carlos I de Física Teórica

y Computacional

Universidad de Granada, E-18071 Granada, Spain

E-mail: earriola@ugr.es

\begin{abstract}
We evaluate the valence double parton distribution (dPDF) of the pion in the Nambu-Jona-Lasinio model. At the low-energy quark-model scale and in the chiral limit a particularly simple factorized form $D\left(x_{1}, x_{2}, \vec{q}\right)=\delta\left(1-x_{1}-x_{2}\right) F(\vec{q})$ follows, where $x_{1,2}$ denote the longitudinal momentum fractions of the valence quark and antiquark, and $\vec{q}$ is their relative transverse momentum. For $\vec{q}=\overrightarrow{0}$ our result complies to the Gaunt-Sterling sum rules. We carry out the necessary dDGLAP evolution to higher scales via the Mellin moments and explore its impact on the correlation defined as the ratio of dPDF to the product of single parton distributions, $D\left(x_{1}, x_{2}, \vec{q}=\overrightarrow{0}\right) / D\left(x_{1}\right) D\left(x_{2}\right)$. Since the ratios of the valence Mellin moments $\left\langle x_{1}^{n} x_{2}^{m}\right\rangle /\left\langle x_{1}^{n}\right\rangle\left\langle x_{2}^{m}\right\rangle$ are invariants of the dDGLAP evolution, they may serve as robust measures of these correlations. Model predictions, which can be tested in the upcoming lattice simulations, are provided. We also discuss the transverse form factor related to the dPDF of the pion.
\end{abstract}

Light Cone 2019 - QCD on the light cone: from hadrons to heavy ions - LC2019

16-20 September 2019

Ecole Polytechnique, Palaiseau, France

\footnotetext{
* Speaker.

$\dagger$ Supported by the Polish National Science Centre (NCN) Grant 2018/31/B/ST2/01022, the Spanish Ministerio de Economia y Competitividad and European FEDER funds (grant FIS2017-85053-C2-1-P) and Junta de Andalucía grant FQM-225.
} 
More details of this talk and complete references are given in [1]. Double parton distributions (dPDFs) have recently drawn considerable attention due to evidence for double parton scattering at the LHC. Model evaluations for the pion, such as ours or similar studies of [2], are useful in the light of the awaited lattice QCD results for related quantities, such as moments of dPDFs [3] or existing two currents correlators [4]. Their simplicity illuminates the intricate features of the formal definitions of these objects.

The spin-averaged dPDF (see [6] and references therein) for the parton species $j_{1,2}$ in a hadron with momentum $p$ is defined via the following forward matrix element,

$D_{j_{1} j_{2}}\left(x_{1}, x_{2}, \vec{y}\right)=\left.2 p^{+} \int d y^{-} \frac{d z_{1}^{-}}{2 \pi} \frac{d z_{2}^{-}}{2 \pi} e^{i\left(x_{1} z_{1}^{-}+x_{2} z_{2}^{-}\right) p^{+}}\left\langle p\left|\mathscr{O}_{j_{1}}\left(y, z_{1}\right) \mathscr{O}_{j_{2}}\left(0, z_{2}\right)\right| p\right\rangle\right|_{z_{1}^{+}=z_{2}^{+}=y^{+}=0, \vec{z}_{1}=\vec{z}_{2}=\overrightarrow{0}}$

where $\vec{y}$ is the transverse distance between the two partons and the light-cone coordinates are $a^{ \pm}=\left(a^{0} \pm a^{3}\right) / \sqrt{2}$. For the case of quarks and antiquarks considered here, the bilocal color-singlet operators are explicitly

$$
\mathscr{O}_{q}(y, z)=\frac{1}{2} \bar{q}\left(y-\frac{z}{2}\right) \gamma^{+} q\left(y+\frac{z}{2}\right), \quad \mathscr{O}_{\bar{q}}(y, z)=-\frac{1}{2} \bar{q}\left(y+\frac{z}{2}\right) \gamma^{+} q\left(y-\frac{z}{2}\right) .
$$

The summation over color is implicit, whereas the flavor indices are skipped. Since the quark and antiquark coordinates within the operators $\mathscr{O}_{j}$ in Eq. (1) are not split in the transverse direction, the light-front gauge along straight line paths sets the Wilson gauge link operators to identity.

Evaluation according to the diagram of Fig. 1 in the chiral limit yields the result of an appealing simplicity,

$$
D\left(x_{1}, x_{2}, \vec{q}\right)=\delta\left(1-x_{1}-x_{2}\right) F(\vec{q}),
$$

holding at a low-energy quark model scale. Moreover, it satisfies the Gaunt-Stirling sum rules [7], which is nontrivial to accomplish in parametrization approaches $[8,9,10]$. We note a factorization of the longitudinal and transverse degrees of freedom, similarly to the case of single parton distributions (sPDFs) of the pion in the NJL model. The result of Eq. (3) befits a simple phase space calculation with $x$-independent matrix elements [11].

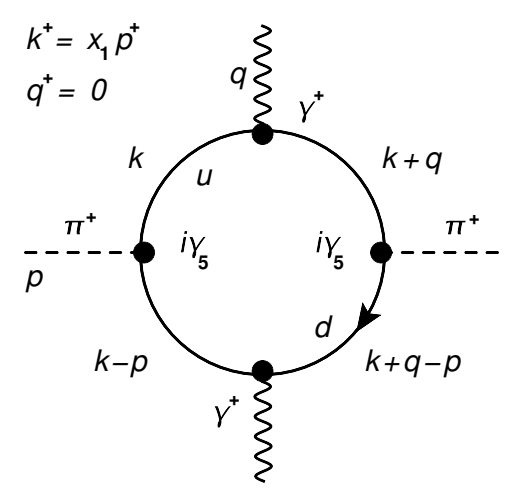

Figure 1: Feynman diagram in momentum space for the evaluation of the valence quark dPDF of the pion in chiral quark models. A suitable ultraviolet momentum cut-off, implemented in a gauge-invariant way via spectral regularization [5] or Pauli-Villars method, is understood. 

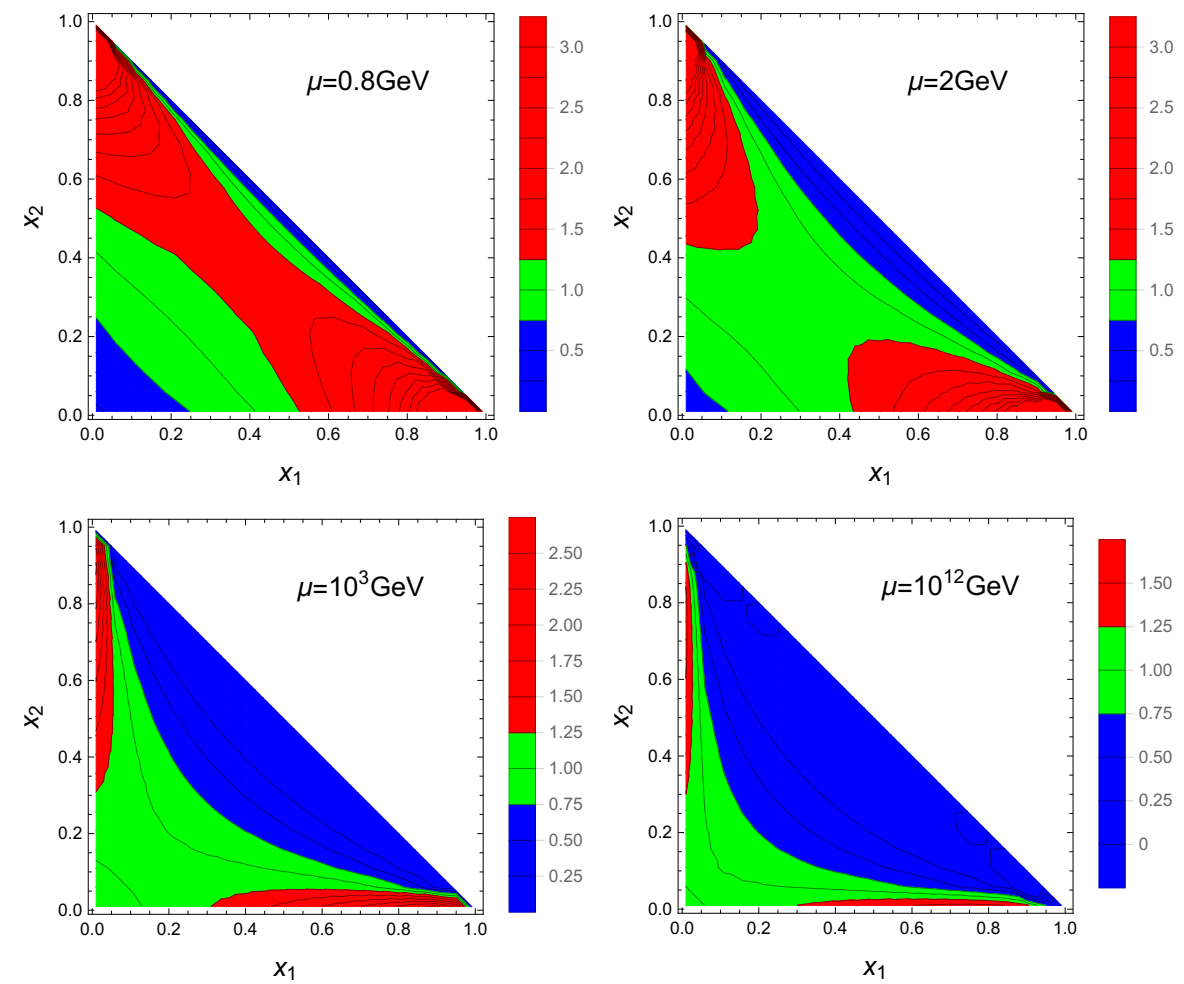

Figure 2: Correlation $D_{u \bar{d}}^{\pi^{+}}\left(x_{1}, x_{2}, \vec{q}=\overrightarrow{0}\right) /\left[D_{u}\left(x_{1}\right) D_{\bar{d}}\left(x_{2}\right)\right]$ at increasing evolution scales $\mu$.

QCD evolution is a necessary ingredient of the phenomenology built on low-energy models, as it allows to bring the results to the energy domain accessible in experiments and lattice simulations. The dDGLAP evolution of the valence Mellin moments is particularly simple, due to the absence of the inhomogeneous term. It immediately follows that the ratios $r_{n m}=\left\langle x_{1}^{n} x_{2}^{m}\right\rangle /\left\langle x_{1}^{n}\right\rangle\left\langle x_{2}^{m}\right\rangle$ for the valence distributions are scale invariant, because the evolution ratios cancel out. The valence Mellin moments of sPDFs and dPDFs evolve as

$$
M_{n}(\mu)=\left(\frac{\alpha(\mu)}{\alpha\left(\mu_{0}\right)}\right)^{\gamma_{n} / 2 \beta_{0}} M_{n}\left(\mu_{0}\right), \quad M_{n m}(\mu)=\left(\frac{\alpha(\mu)}{\alpha\left(\mu_{0}\right)}\right)^{\gamma_{n} / 2 \beta_{0}+\gamma_{m} / 2 \beta_{0}} M_{n m}\left(\mu_{0}\right),
$$

with $\gamma_{i}$ denoting the appropriate anomalous dimensions, which leads to the advocated invariance of $r_{n m}$. This very simple feature has far-reaching consequences, as it brings an insight into the nonperturbative dynamics at low energy scales from the data at high energy scales. In the NJL model we find from Eq. (3) $r_{n m}=(1+n) !(1+m) ! /(1+n+m)$ !. The largest ratio is for $m=n=1$. The particular set of values for the moments is specific to a given low-energy model. The LO dDGLAP evolution of the lowest Mellin moments of the valence dPDF of the pion is shown in Fig. 3. Of course, the fall-off with the scale $\mu$ is controlled by the appropriate anomalous dimensions in Eq. (4).

The dPDF form factor in the transverse variable both in momentum space as well as in coordinate space is shown in Fig. 4 for both the NJL model, PV regularized [12] and the Spectral Quark Model (SQM) [5]. The form factor can be shown [1] to mathematically correspond to a convolution of a wave function with itself. A remarkable feature seen from Fig. 4 is the lack of positivity, 


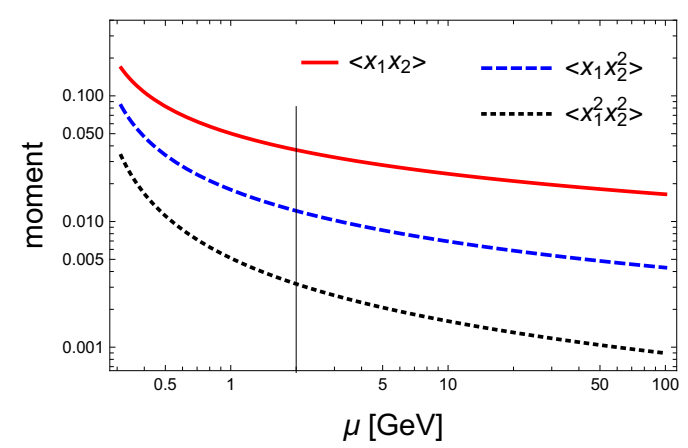

Figure 3: Lowest Mellin moments $M_{n m}$ of the valence dPDF of the pion plotted as functions of the evolution scale $\mu$.

which is surprising but does not contradict any general requirement. For instance, a convolution of functions with nodes with itself is not necessarily positive. Future lattice QCD calculations may shed some light on this interesting problem.

Finally, we comment on possible lattice determinations of quantities related to dPDFs. Firstly, on the lattice the currents are local, with $z_{1}^{-}$and $z_{2}^{-}$in Eq. (1) set to zero, which corresponds to the integration over $x_{1}$ and $x_{2}$. Second, the relative time difference of the two currents in $[4,3]$ is set to zero, whereas in Eq. (1) unconstrained integration over $y^{-}$is carried out. Thus, our dPDF results for the form factors cannot be directly compared to $[4,3]$, even if the pion mass were taken to its lattice value of $\sim 300 \mathrm{MeV}$.

To summarize our main results:

- The chiral quark models yields in the chiral limit a factorization of the longitudinal and transverse dynamics. At the low-energy quark model scale, the longitudinal distribution is
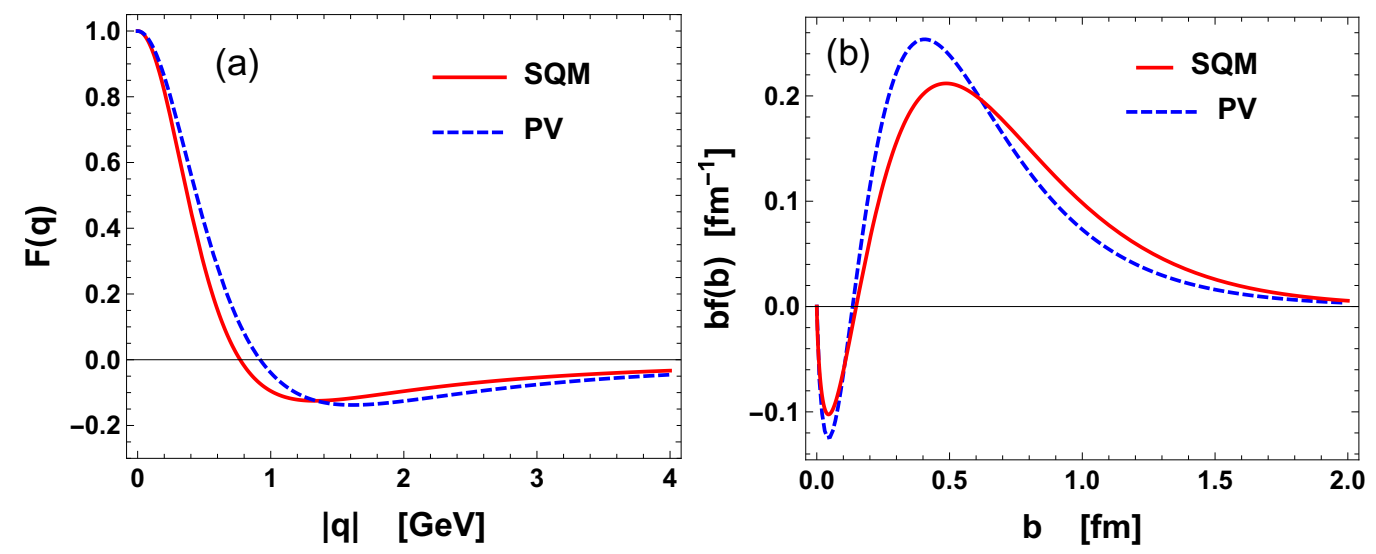

Figure 4: dPDF form factor in the momentum space, $F(\vec{q})$, (left panel) and coordinate space, $b f(b)$, (right panel), plotted as a functions of the corresponding transverse variable for the NJL model with the PV regularization and for SQM. The form factors $F(\vec{q})$ and $f(\vec{b})$ are connected by a Fourier-Bessel transform. 
just $\delta\left(1-x_{1}-x_{2}\right)$, as follows from the momentum conservation.

- We have carried out the LO dDGLAP evolution via the Mellin moments.

- The Gaunt-Stirling sum rules are explicitly satisfied in our approach.

- The longitudinal correlation function, defined as the ratio of dPDF from the product of two sPDFs, allows for a test of the validity of the commonly used product ansatz. The conclusion is that at high $\mu$ and small momentum $x_{1}$ and $x_{2}$ it is justified within the present experimental kinematics and accuracy.

- The $r_{n m}$ moments, which are invariants of the evolution, are particularly useful objects for future lattice studies, as they allow to look down into low energies and scrutinize nonperturbative aspects of the pion structure.

- The dPDF form factor becomes negative above large transverse momenta $\sim 0.8 \mathrm{GeV}$ or below transverse separations $\sim 0.15 \mathrm{fm}$.

\section{References}

[1] W. Broniowski and E. Ruiz Arriola, Double parton distribution of valence quarks in the pion in chiral quark models, 1910.03707.

[2] A. Courtoy, S. Noguera and S. Scopetta, Double parton distributions in the pion in the Nambu-Jona-Lasinio model, JHEP 12 (2019) 045 [1909. 09530 ].

[3] RQCD collaboration, Double Parton Distributions of the Pion, PoS LATTICE2016 (2016) 152 [1701.05479].

[4] G. S. Bali, P. C. Bruns, L. Castagnini, M. Diehl, J. R. Gaunt, B. Gläßle et al., Two-current correlations in the pion on the lattice, JHEP 1812 (2018) 061 [1807.03073].

[5] E. Ruiz Arriola and W. Broniowski, Spectral quark model and low-energy hadron phenomenology, Phys. Rev. D67 (2003) 074021 [hep-ph/ 0301202 ].

[6] M. Diehl, D. Ostermeier and A. Schafer, Elements of a theory for multiparton interactions in QCD, JHEP 1203 (2012) 089 [1111.0910].

[7] J. R. Gaunt and W. J. Stirling, Double Parton Distributions Incorporating Perturbative QCD Evolution and Momentum and Quark Number Sum Rules, JHEP 1003 (2010) 005 [0 910 . 4347].

[8] W. Broniowski and E. Ruiz Arriola, Valence double parton distributions of the nucleon in a simple model, Few Body Syst. 55 (2014) 381 [1310 . 8419].

[9] K. Golec-Biernat, E. Lewandowska, M. Serino, Z. Snyder and A. M. Stasto, Constraining the double gluon distribution by the single gluon distribution, Phys. Lett. B750 (2015) 559 [1507. 08583 ].

[10] W. Broniowski, E. Ruiz Arriola and K. Golec-Biernat, Generalized Valon Model for Double Parton Distributions, Few Body Syst. 57 (2016) 405 [1602 . 00254 ].

[11] E. Ruiz Arriola, On the structure functions of mesons and baryons in a chiral quark model, in Hadrons as solitons: Proceedings, Mini-Workshop, Bled, Slovenia, July 6-17, 1999, pp. 5-16, 1999, hep-ph/9910382.

[12] E. Ruiz Arriola, Pion structure at high and low energies in chiral quark models, Acta Phys. Polon. B33 (2002) 4443 [hep-ph/ 0210007$].$ 